\title{
La place des outils numériques dans l'enseignement du projet: comparaison entre une école française et une école américaine
}

\section{Digital tools in architecture design education: A comparison between a French and American models}

\author{
Laurent Lescop $^{1, *}$ et Olivier Chamel $^{2}$ \\ ${ }^{1}$ ENSA Nantes AAU-CRENAU, UMR-CNRS 1563 - Nantes, France \\ ${ }^{2}$ FAMU, Tallahassee, Floride, EU
}

\begin{abstract}
Résumé. Un quart de siècle est passé depuis la généralisation de l'informatique graphique dans les écoles d'architecture, une génération d'étudiants est passée et pourtant, les mêmes questions animent les équipes pédagogiques sur la place à donner aux outils, à leur place et l'influence qu'ils peuvent avoir dans la conception et dans la représentation. Nous proposons ici une grille d'analyse conceptuelle et pédagogique afin de faire un état des lieux et tenter un point d'étape à ce débat.
\end{abstract}

Mots-clés. Pédagogie du projet, Conception numérique, comparaison France-USA, prospective.

\begin{abstract}
A quarter of a century has passed since the generalization of computer graphics in schools of architecture, a generation of students has passed and yet the same questions animate the teaching teams on the place to give to the tools, their role and the impact they can have in design and representation. We propose here a grid of conceptual and pedagogical analysis in order to take stock of the situation and to attempt a step forward in this debate.
\end{abstract}

Keywords. Pedagogy, Design process, Digital tools, France-US comparison, prospective.

\section{Avant-propos}

La première version de cet article a été écrite en décembre 2019 alors que le covid19 n'était qu'une information parmi d'autres en provenance de Chine. Quatre mois plus tard, le monde est paralysé, les écoles et universités sont fermées, les étudiants et les enseignants

\footnotetext{
*1aurent.lescop@nantes.archi.fr
} 
sont confinés chez eux. La conséquence en a été un basculement rapide en mode «virtuel» pour assurer la continuité pédagogique et la recherche de moyens plus souples, plus interactifs pour maintenir le lien pédagogique avec les étudiants. Avant le confinement, l'idée de dématérialiser la pédagogie avait été exprimée dans notre école et quelques projets étaient vaguement à l'étude pour la réalisation de MOOC, les Massive Open Online Course. Pris de cours, nous créons des contenus pour lesquels nous passons un temps considérable. L'épreuve des faits commence à porter des questionnements passionnants pour cerner la nouvelle place des outils numériques dans la pédagogie. Si d'un côté, la dématérialisation permet lors du confinement une paradoxale ouverture au monde avec la possibilité «d'inviter» des collègues lointains à participer aux projets, ce, sans d'autres coûts que ceux de la connexion, cette même dématérialisation rend plus évidente les inégalités devant l'équipement des étudiants. Si ces derniers possèdent de bonnes machines et un accès aux réseaux rapide, le travail n'en devient que plus fluide et interactif, dans le cas contraire, à l'isolement s'ajoute la fatalité de ne pouvoir produire. Cette épreuve du covid19, au-delà de l'utilisation même des outils qui sera discutée ici, nous invite à prendre en compte un contexte beaucoup plus large, qui décrit les conditions d'accès avant même les conditions d'usage.

\section{Crayon ou souris ? Déplacement des enjeux}

La question de la place des outils numériques est celle que l'on aime encore éviter dans les débats pédagogiques. La sentence «ce n'est qu'un outil» disqualifie de fait des interrogations plus profondes que nous devons, en tant qu'enseignants, explorer avant même de nous présenter aux étudiants. Les outils étant omniprésents, leur légitimité ne semble plus être un sujet de discussion et en même temps, la complexité de leur maitrise à un niveau supérieur exclu de fait les non experts d'une évaluation pertinente.

Il faut donc changer d'axe d'analyse, pour faire évoluer le débat, ne prenons paradoxalement plus le numérique comme point d'entrée des réflexions que nous pouvons porter sur la pédagogie mais comme un des indicateurs ou un des traceurs de la pédagogie proposée aux étudiants. Dans le processus qui va de la question et de l'exposé des objectifs à leur réalisation par les étudiants, le numérique se manifeste comme un indicateur de complexité que l'on va retrouver, par exemple, dans le calcul des structures, dans les réponses environnementales, dans l'engendrement des formes ou encore dans la prise en compte d'un ensemble de données contextuelles politiques ou sociales.

En d'autres termes, l'évaluation du renouvellement ou non des paradigmes de conception, bien plus que ceux de la représentation, passe par l'analyse fine des étapes de maturation du projet et la façon dont nous enseignons ces étapes aux étudiants niveau par niveau. Les attentes que nous formulons pour la conception prédit le recours ou non au numérique et les démarcations que nous opérons d'avec les méthodes analogiques. Si AutoCad n'a pas fondamentalement modifié les modes de représentation du projet et si le paramétrique doit beaucoup à D'Arcy-Thomson, il parait toutefois difficile de ne pas lier outils et types de production.

D'autant qu'en 20 ans, nous avons effectué, parfois à notre corps défendant, deux bascules dans le monde de l'architecture, deux bascules qui ont fortement perturbé l'organisation du monde des agences : la première a été celle de l'informatisation des agences au passage de l'an 2000 et la deuxième est celle du BIM dix ans plus tard. Une nouvelle bascule se profile, plus radicale encore, c'est celle de l'Intelligence Artificielle qui permettra une production automatisée des tâches basiques de conception puis d'exécution des pièces graphiques. Autodesk a déjà bien avancé sur ce terrain avec son approche "generative design» (https://www.autodesk.com/solutions/generative-design) et le projet Dreamcatcher (https://autodeskresearch.com/projects/dreamcatcher). L'agence Finch en 
Suède (finch3d.com) avance régulièrement sur la mise au point d'outils génératifs automatisant les taches identifiées comme étant de la conception. Cela concerne par exemple l'optimisation de plans de logements ou même l'implantation de bâtiments sur une parcelle. Nous pouvons même trouver des exemples de conception optimisées basées sur l'usage comme celle proposée par l'Université Technion (Schaumann\& al, 2017).

En posant la question sous une forme dialectique crayon ou souris, l'idée est d'évaluer l'anticipation ou la résistance que nous pouvons avoir face à des outils et pour faire apparaitre les traceurs évoqués plus tôt, nous établirons un tableau croisé des thématiques de conception, de leur apparition dans le cursus et de l'utilisation du numérique pour élaborer des réponses dont il sera possible d'évaluer la complexité. Nous poserons auparavant quelques jalons conceptuels qui aideront à contextualiser le propos.

\section{Problème: enjeux des outils numériques dans la formation des architectes, exemple et comparaison ensa Nantes et FAMU Tallahassee.}

La question du positionnement des outils numériques dans la formation des architectes anime depuis longtemps chaque école (Violeau, 1999). La démarche que nous présentons ici est une comparaison entre deux écoles dont les programmes sont en cours de redéfinition pour le renouvellement des accréditations. Il s'agit d'un côté de l'Ecole Nationale Supérieure d'Architecture de Nantes en France et de l'autre de la Florida A\&M University (FAMU) à Tallahassee en Floride aux Etats-Unis. Avant la crise du covid19, les deux écoles étaient dans des contextes économiques très différents, avec en France un marché assez contraint et des agences plutôt de petite taille selon l'observatoire de la profession et aux Etats-Unis, un marché très dynamique avec des agences comprenant de nombreux salariés et collaborateurs. Cette différence a un impact fort sur la pédagogie avec d'un côté une obligation presque vitale d'exploiter des marchés de niche et de l'autre de se conformer à la demande du marché.

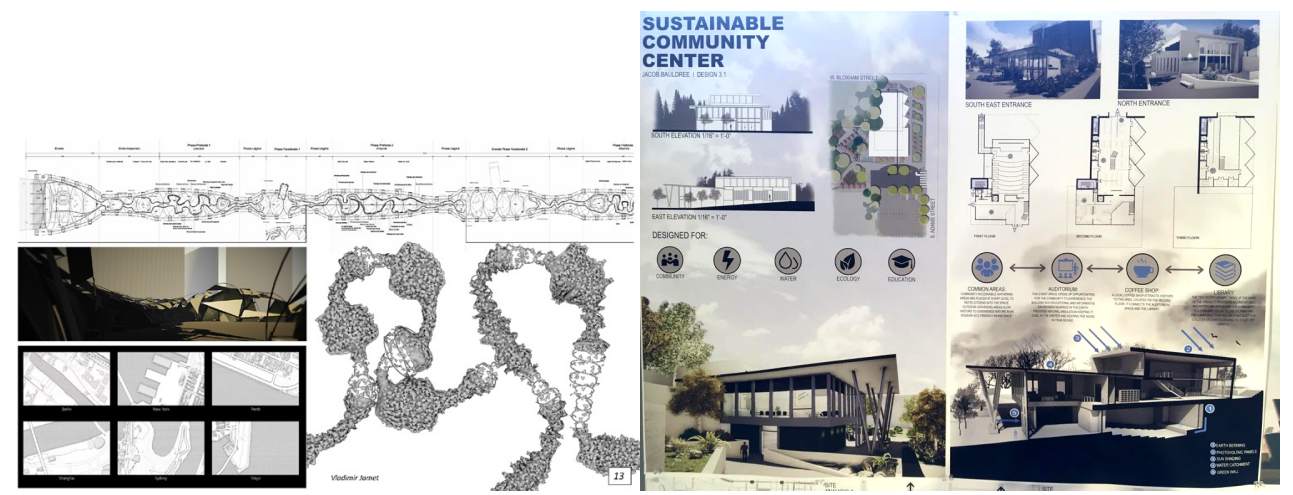

Figure 1. Vladimir Jamet, Master 2, ensa Nantes, génération paramétrique de plans architecturaux. Jacob Bauldree, Licence3, FAMU Tallahassee, conception éco-responsable

\subsection{Question de recherche : héritage et émergence}

Autour de la notion de projet, le principal marqueur identitaire de la culture architecturale, une importante nuance doit être marquée entre le savoir et le savoir-faire, entre la réflexion que l'on élabore sur une pratique et la pratique elle-même. Les enseignements des écoles d'architecture articulent les dimensions théoriques et pratiques, 
toutefois, il existe toujours un rapport ambigu aux concepts et processus nouveaux que peuvent introduire, par exemple, les outils numériques. L'appartenance de la profession à des ordres et l'embarras toujours présent à identifier une recherche propre en architecture qui cherche à exister dans le monde académique, nous conduit à proposer, pour éclairer la suite de notre proposition, un modèle de compréhension de nos enjeux pédagogiques selon deux modalités, la modalité de l'héritage et la modalité de l'émergence.

\subsection{La modalité de l'héritage}

La modalité de l'héritage porte la transmission d'un contenu et s'inscrit dans une continuité. La notion d'héritage, que l'on va comprendre ici comme l'héritage professionnel, scientifique et technique, est construit comme une succession de périodes interdépendantes qui chacune cherchent à épuiser un paradigme théorique ou technique. En architecture existent deux modalités de l'héritage. La première est celui de la profession qui par la pratique et par les écoles assure la transmission d'un contenu et d'un protocole qui intègre l'origine du contenu et la façon dont les contenus ultérieurs doivent se constituer et s'organiser. Il est intéressant de noter que dans la volonté d'identifier une recherche architecturale propre, cette dernière a été intégrée à une deuxième modalité de l'héritage, celle de la recherche académique universitaire, qui définit et organise, la production des enseignants chercheurs. On attache, à cette transmission, certaines valeurs, disciplinaires et scientifique qui justifieront d'écarter de cette transmission des éléments ne permettant pas la conservation du sens préalable. C'est surtout une transmission qui s'inscrit dans le maintien et la défense d'une légitimité qui ne s'acquiert que par la maitrise des codes et la reconnaissance des pairs. Il faut donc des autorités qui non seulement perpétueront cette modalité mais également la feront fonctionner comme une téléologie, c'est-à-dire avec une finalité qui est, évidemment, la préservation et la transmission d'un statut. L'ordre des Architectes, les institutions académiques, la formation et la validation des connaissances s'inscrivent dans ce récit avec la volonté d'atteindre un registre d'universalité. Il existe une forme de transcendance dans cette approche avec la création de symboles et des rites que le public va reconnaitre afin de constituer une adhésion sociale à l'autorité professionnelle et scientifique. Il est particulièrement intéressant de voir ainsi la pratique et la recherche unies dans cette modalité de l'héritage alors qu'ils sont souvent sur des terrains d'opposition.

\subsection{La modalité de l'émergence}

Thierry Verdier écrit: "l'architecture, par l'infini des savoirs et des compétences qu'elle représente, ne peut se circonscrire dans le cadre étroit d'une discipline académique ou universitaire. Peut-on admettre que le doctorat en architecture soit contraint de se soumettre aux grands cadres scientifiques et étanches distinguant les disciplines universitaires entre elles? Certainement pas.» Et de rappeler que «le risque est grand de croire que lorsque l'architecte fait un projet, il fait une recherche. Non, il exerce simplement son métier. La recherche en architecture, c'est précisément dépasser le métier pour, peu à peu, interroger la fabrication de cette pensée complexe. (Verdier 2014) Cela implique d'inventer des modes de recherche propres, d'enseignement qui ne font ni l'impasse sur la rigueur de la démarche et encore moins sur les ambitions intellectuelles.

C'est ce mouvement que l'on pourrait qualifier, en contraste avec la modalité de l'héritage, modalité d'émergence. La modalité d'émergence ne fonctionne pas sur le principe de succession comme c'est le cas pour la modalité de l'héritage, il n'est pas sur un principe linéaire. C'est une modalité qu'il faut effectuer, c'est-à-dire, activer. Une fois activée, elle peut, mais surtout elle doit, témoigner de la permanence de ce qui est reconnu comme tel. L'autorité qui active la modalité lui reconnait son origine et donc valide son 
authenticité. En France, cela pourrait être le réseau des laboratoires de recherche et les institutions qui évaluent la valeur de ce qui est produit. La modalité d'émergence cherche à s'attacher une fidélité à l'origine, en l'occurrence à la production du projet mais aussi aux canons académiques, ce qui se traduit par la recherche d'une expérience holistique, c'est-àdire totale. En définissant la recherche architecturale comme impliquée, il s'élabore un rapport dialectique entre la recherche en architecture et la société dans laquelle elle s'exerce. Ce rapport dialectique est celui que l'on doit entretenir pour répondre aux questions contemporaines, identifier la naissance de nouvelles pratiques et peut-être aussi paradoxalement, par la poursuite d'une plénitude holistique et préserver la valeur de l'architecte dans sa propre pratique.

C'est donc bien dans une modalité d'émergence que nous devons observer les effets d'influence et les modalités d'application des idées et des outils que le monde numérique fait circuler autour de nous.

\section{Hypothèse de travail}

Notre horizon de travail étant identifié, il convient maintenant de se dégager des «attracteurs» thématiques qui brouillent la vision globale du propos. Nous ne voulons pas entrer dans les considérations mettant en concurrence le couple main/cerveau avec la machine et développer autour d'une concurrence homme/machine dans l'acte de conception. Le sujet est largement traité ailleurs et devient stérile à ne pas chercher à définir ce que l'on cherche à concevoir. Notre propos, nous le verrons ensuite est de définir les besoins conceptuels et techniques rythmant le projet et d'observer à quels moments la machine devient incontournable. Le deuxième attracteur, lié au premier, est la primauté du dessin dans l'acte de conception. Jean-François Coulais rappelle que «on considère aujourd'hui trop souvent la pratique du dessin et le processus de conception architecturale comme indissociables, et ce depuis l'antiquité. Or ce postulat, issu de l'histoire de la théorie et des styles architecturaux, doit être largement nuancé à la lecture des faits apportés par l'archéologie et l'histoire médiévale d'une part, par l'histoire des sciences et des techniques de l'autre »(Coulais 2014, p.75). Evoquons donc à ses côtés de visibilités calculées même si le faisons en pleine conscience d'une forme de déterminisme culturel (Lescop - Lu, 2018). Le troisième attracteur est celui qui considère le logiciel comme un simple outil ne prédéterminant ni la façon de concevoir et encore moins le résultat produit. Il n'est qu'à voir la sélection 2020 du magazine AD pour se faire une opinion que ce soit le siège du groupe de presse Axel Springer par OMA à Berlin, le Musée Atelier Audemars Piguet par Bjarke Ingel BIG dans la vallée de Joux en Suisse ou encore le Nanjing Zendai Himalayas Center par MAD Architects et Ma Yanso à Nanjing en Chine pour s'en convaincre. Mais là encore, il ne faut pas confondre le résultat ou le style avec le processus d'engendrement ou de conception. Il ne faut pas non plus généraliser à partir de productions exceptionnelles alors que la production quotidienne ne lui ressemble pas. Il est cependant hasardeux de penser que l'outil et par extension les matériaux, n'ont pas d'influence sur le processus de conception et le résultat produit. Le dernier attracteur duquel nous devons nous méfier est le BIM comme synecdoque du numérique et parfois même de la production architecturale. Nous l'avons évoqué plus haut, le BIM définit un nouveau franchissement dans la pratique du projet, l'organisation des agences et la définition de nouvelles compétences indispensables à la bonne conduite d'une opération. Le BIM réplique le choc de l'informatisation des agences juste avant l'an 2000 avec les même phénomènes de résistances et d'adoptions forcées (Hochscheid - Halin, 2018)

Pour dresser notre état des lieux, nous sommes partis sur un inventaire des tâches, qu'en pédagogie l'on va reconnaitre comme étant des compétences, importantes à mobiliser pour analyser, imaginer, concevoir et fabriquer un projet architectural. Pour chacune de ces 
tâches, nous avons repéré à quel moment elles apparaissent dans le programme des écoles de Nantes et de Tallahassee. Nous nous sommes ensuite interrogés sur la récurrence de la tâche ou dit autrement sa mobilisation ponctuelle ou structurelle et enfin sur l'utilisation ou pas d'un outil numérique pour réaliser cette tâche. Deux groupes se sont assez naturellement distingués, un groupe mettant en jeu la représentation et la fabrication et un autre groupe impliquant la manipulation des données.

\subsection{Tableaux synoptiques}

\subsubsection{Méthodologie}

Pour réaliser cette étude, nous avons commencé par faire un inventaire des cours disponibles par année, par matière. Nous avons de plus interrogé les collègues enseignants. Pour la FAMU, le travail a été facilité du fait que l'un des coauteurs de cet article est également directeur des programmes. Pour le second coauteur, il a été nécessaire d'interroger les collègues et les confirmations sont venues de documents circulant pour la rédaction du rapport HCERES (Haut Conseil de l'évaluation de la recherche et de l'enseignement supérieur) qui permettra l'évaluation de l'école.

Les deux écoles fonctionnent de façon différentes quant à la conception des programme et à leur application. A la FAMU, le comité de direction décide de l'orientation des programmes et distribue ensuite les cours aux enseignants. Il existe bien entendu une marge de négociation et une adéquation aux qualifications de chacun, mais le système est malgré tout de nature «top-down ». La maîtrise des contenus est donc bien assurée. Pour l'ensa Nantes, le programme se fait sur proposition des enseignants qui soumettent au conseil pédagogique et scientifique (CPS) et plus particulièrement à la commission des formations et de la vie étudiante (CFVE) qui en est l'une des composante avec commission de la recherche (CR).

Pour la FAMU, l'inventaire des logiciels est relativement facile à établir car il est très encadré. Le choix est effectué par la direction des études et le déploiement se fait ensuite. La typologie des rendus, est également relativement uniformisée et réponds aussi à des compétences réclamées sur le marché du travail, ce, même très tôt dans les études. On voit donc apparaitre les couples Formit / Illustrator dès les premiers semestres, puis Rhino / Revit dans la suite du cursus. En ajoutant Enscape, nous avons les outils très majoritairement mobilisés à Tallahassee. Pour l'ensa Nantes, du fait de la taille de l'école et de la ventilation de l'apprentissage des outils entre ateliers, cours spécialisés et autoformation, l'inventaire est beaucoup plus large. Il part d'une base commune Sketchup, Autocad, puis se diversifie avec Revit et Archicad, et intègre Twinmotion et Lumion pour les rendus et le temps réel. A cela s'ajoute Grasshopper dès la $2^{\text {ème }}$ année et pléthore de logiciels spécialisés pour les calculs de structure, de thermiques et pour la cartographie. La maitrise des outils informatique est réputée comme plutôt très bonne à Nantes.

Les thématiques du projet au sens large ont été inventoriées puis organisées par années et par différentiation progressive de ce qui peut être réalisé de façon identique à la main de ce qui réclame exclusivement l'usage de logiciels dédiés. Cet inventaire a progressivement laissé apparaitre deux familles, l'une répondant assez bien aux modalités de l'héritage, tandis que l'autre illustre les modalités de l'émergence.

\subsubsection{Tableau 1 : Représentation, Fabrication-Modalités de l'héritage}

Ce que met en avant ce premier tableau, c'est comment chacune des tâches peut être réalisée soit de façon analogique soit de façon numérique. Les deux options ne sont plus 
aujourd'hui concurrentes dans les écoles mais concourantes, laissant le choix à l'étudiant d'utiliser un média ou un autre selon sa dextérité, l'adéquation au projet ou les conseils de l'enseignant. Seule la pratique de la réalité virtuelle appartient purement au monde numérique et peut faire réellement schisme. A Nantes, elle est marginale et très tardivement enseignée. A Tallahassee, il va être question de l'introduire dès la 1 ère année prenant comme postulat de base que les étudiants intégrant l'école sont déjà très familiarisés avec ces supports et techniques via le monde du jeu vidéo. Introduire la réalité virtuelle ou augmentée dès la première année bouleverse tous les discours sur la relation main / outil / cerveau puisque l'on est désormais capable de concevoir, par gestes naturels, dans un espace de représentation, des formes, des organisations, ce, à toutes les échelles et en ayant visuellement et presque physiquement le résultat de chacune des décisions.

Tableau 1. Représentation, Fabrication-Modalités de l'héritage.

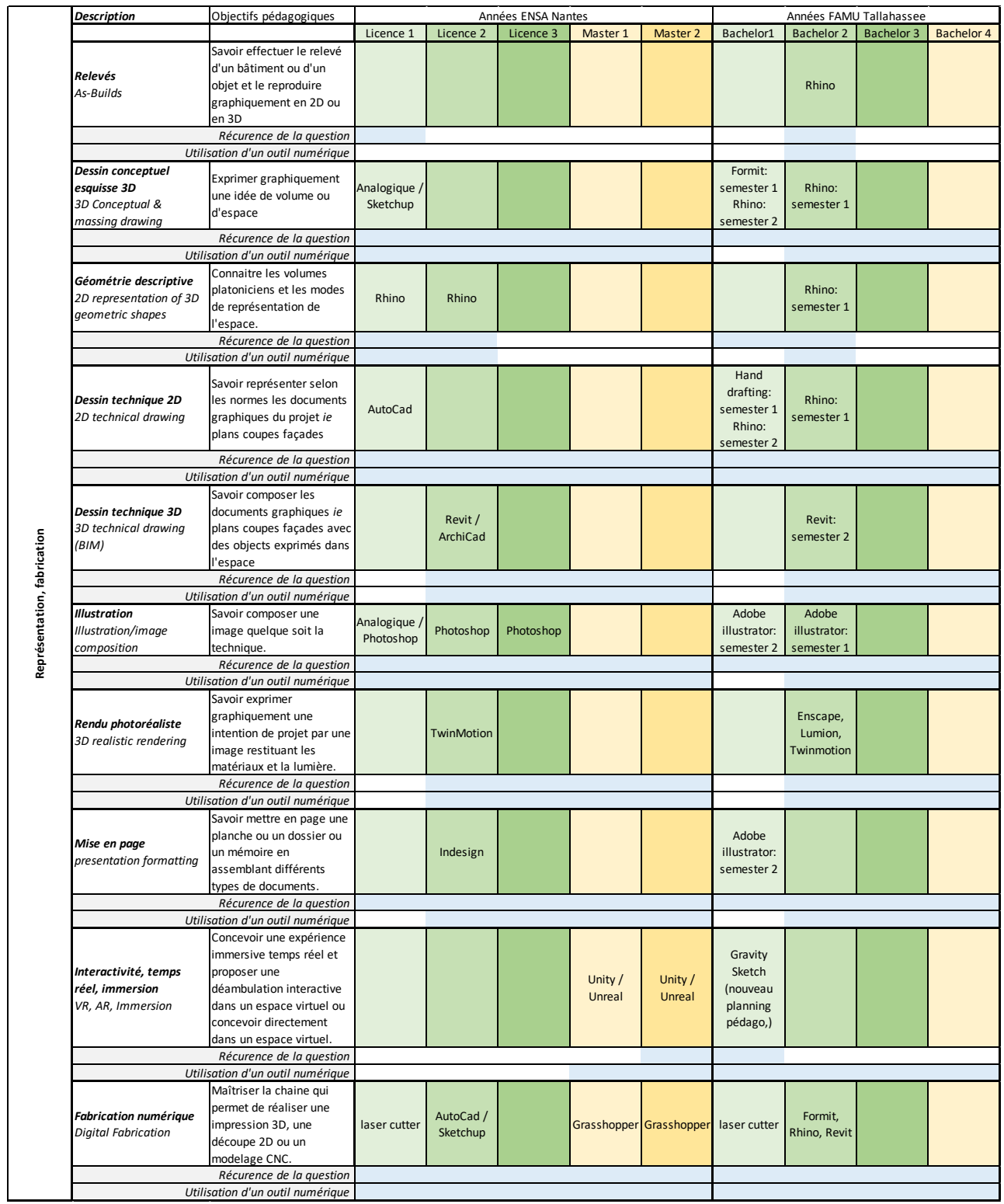


Le premier tableau montre en tout cas un très bon ancrage des compétences lorsqu'elles sont proposées dans le cursus et leur pérennité au long de la formation. Mise à part les relevés et la géométrie descriptive, les compétences sont reconnues comme indispensables.

\subsubsection{Tableau 2 : Identification, gestion, importation, exploitation de données, modalités de l'émergence.}

Le tableau 2 met en valeur les enjeux réels du numérique dans la pédagogie et la pratique du projet. Toutes les tâches identifiées ici sont d'une part dépendantes de données qu'il faut identifier et organiser et d'autre part qui demandent de lourds traitements pour les intégrer aux projets. Il existe deux grandes façons de réaliser les tâches qui sont listées dans le tableau : soit elles interviennent en aval de la réflexion formelle et viennent corriger les manquements du projet en termes d'acoustique, d'ensoleillement, de statique etc...; soit viennent en amont de la réflexion ou sont intiment intégrées à la réflexion pour la mise au point des formes. Dans le deuxième cas, la complexité et la masse des données à traiter simultanément rend indispensable le recours à la puissance informatique pour identifier non pas la solution, mais un ensemble de solutions dans lesquelles le concepteur fera son choix.

Tableau 2. Identification, gestion, importation, exploitation de données, modalités de l'émergence.

\begin{tabular}{|c|c|c|c|c|c|c|c|c|c|}
\hline \multirow{24}{*}{ 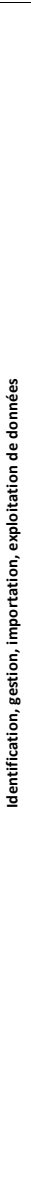 } & $\begin{array}{l}\text { Gestion de données } \\
\text { Data mining and } \\
\text { management }\end{array}$ & $\begin{array}{l}\text { Savoir trouver, identifier, } \\
\text { fiabiliser, trier, organiser } \\
\text { et importer de la donnée / } \\
\text { savoir exporter de la } \\
\text { donnée }\end{array}$ & & & & & & & \\
\hline & & Récurence de la question & & & & & & & \\
\hline & Utili & sation d'un outil numérique & & & & & & & \\
\hline & $B I M$ & $\begin{array}{l}\text { Organiser son travail et } \\
\text { ses données de façon à } \\
\text { favoriser l'interpolarité } \\
\text { entre logiciels. }\end{array}$ & & $\begin{array}{l}\text { Revit/ } \\
\text { Archicad }\end{array}$ & & & $\begin{array}{c}\text { Revit: } \\
\text { semester } 2\end{array}$ & & \\
\hline & & Récurence de la question & & & & & & & \\
\hline & Utili: & sation d'un outil numérique & & & & & & & \\
\hline & $\begin{array}{l}\text { Calcul Structures } \\
\text { Structural calculations }\end{array}$ & $\begin{array}{l}\text { Savoir calculter une } \\
\text { structure selon les } \\
\text { matériaux choisis }\end{array}$ & & & & & & & \\
\hline & & Récurence de la question & & & & & & & \\
\hline & Utilit & sation d'un outil numérique & & & & & & & \\
\hline & $\begin{array}{l}\text { Simulations solaires } \\
\text { Daylighting analysis }\end{array}$ & $\begin{array}{l}\text { Savoir évaluer les apports } \\
\text { solaires et modifier le } \\
\text { projet en fonction des } \\
\text { résultats, savoir optimiser } \\
\text { une forme en fonction des } \\
\text { apports solaires. }\end{array}$ & Sketchup & & Sketchup & & & $\begin{array}{c}\text { Revit: } \\
\text { semester } 2\end{array}$ & \\
\hline & & Récurence de la question & & & & & & & \\
\hline & Utili : & sation d'un outil numérique & & & & & & & \\
\hline & $\begin{array}{l}\text { Simulations Energétique } \\
\text { / Energy analysis }\end{array}$ & \begin{tabular}{|l|} 
Savoir évaluer les apports \\
énergétiques/climatiques \\
et modifier le projet en \\
fonction des résultats, \\
savoir effectuer un bilan.
\end{tabular} & & Solene & & & & & \\
\hline & & Récurence de la question & & & & & & & \\
\hline & Utili & sation d'un outil numérique & & & & & & & \\
\hline & $\begin{array}{l}\text { Simulation acoustique } \\
\text { Accoustic analysis }\end{array}$ & $\begin{array}{l}\text { Comprendre les principes } \\
\text { de diffusion, savoir les } \\
\text { évaluer et intégrer le } \\
\text { phénomène à la } \\
\text { conception. }\end{array}$ & & & $\begin{array}{c}\text { Catt } \\
\text { Acoustique }\end{array}$ & & & & \\
\hline & & Récurence de la question & & & & & & & \\
\hline & Utili & sation d'un outil numérique & & & & & & & \\
\hline & $\begin{array}{l}\text { Cartographie GIS } \\
\text { GIS, mapping }\end{array}$ & $\begin{array}{l}\text { Créer une carte } \\
\text { sémantique intégrant de } \\
\text { I'information à de } \\
\text { multiples échelles, créer } \\
\text { des liens entre les objets. }\end{array}$ & & QGIS & QGIS & & & & \\
\hline & & Récurence de la question & & & & & & & \\
\hline & Utili : & sation d'un outil numérique & & & & & & & \\
\hline & \begin{tabular}{|l} 
Conception \\
paramétrique \\
Parametric design
\end{tabular} & \begin{tabular}{|l} 
Créer une carte \\
sémantique intégrant de \\
l'information à de \\
multiples échelles, créer \\
des liens entre les objets.
\end{tabular} & & & Grasshopper & Grasshopper & & & \\
\hline & & Récurence de la question & & & & & & & \\
\hline & Utili : & sation d'un outil numérique & & & & & & & \\
\hline
\end{tabular}


Ce qui est intéressant à observer, c'est que ces tâches posent deux problèmes liés. Le premier est la compétence de l'étudiant pour la réaliser. Une fois cette compétence acquise on pourrait imaginer qu'elle se maintiendra comme celles identifiées dans le tableau 1. Or, ce n'est que rarement le cas car, et c'est le deuxième problème, il faut des compétences également pour évaluer sa bonne réalisation. Et ces compétences d'évaluation sont doubles puisqu'il faut pouvoir évaluer la bonne conduite du processus (la question est-elle bien posée, les données sont-elles bien identifiées, les résultats couvrent-ils un domaine pertinent) et il faut pouvoir évaluer l'ensemble des solutions proposées et éventuellement celle qui a été choisie. On voit dès lors que les questions relavant du travail sur la donnée, soit pour opérer des simulations, soit pour donner du sens à la collecte d'information touche au sujet de l'objectivation du sensible et de la subjectivisation de la valeur chiffrée.

Le second tableau met également en évidence l'émergence d'un univers culturel dont les références intègrent les outils numériques comme structurant une démarche propre (Nÿs, 2018). La démarche conceptuelle n'est pas forcément liée à la pratique d'un outil mais d'une forme de pensée algorithmique pouvant se développer indépendamment d'une production formelle numérique. Par retour, cette pensée commence à irriguer des formes de pratiques analogiques de construction principalement, ce qui demandera au pédagogue et à l'observateur, de ne pas confondre matérialité de la construction et pensée algorithmique qui la fait naitre et l'accompagne (Leach, 2009).

\section{Conclusion}

Notre analyse, qui va maintenant s'étendre à d'autres écoles en et hors Europe, tente de montrer que les réflexions sur la place du numérique dans la pédagogie des écoles d'architecture et par extension dans les pratiques de l'architecture, si l'on admet que l'architecte peut s'épanouir dans les pratiques émergeantes, est dans l'identification de tâches nouvelles ou complexes pour lesquelles le recours à la machine est peu discutable. Ces tâches ont longtemps été abandonnées à des experts qui petit à petit ont érodé la position hégémonique de l'architecte pour l'encadrer, comme c'est le cas dans le modèle anglo-saxon, entre l'ingénieur structure et l'ingénieur économiste. Or, pour certaines d'entre elles comme la compréhension des phénomènes météorologiques ou énergétiques, ou encore l'économie de la construction, ce sont directement les enjeux d'environnement durable qui sont mis en cause.

A la FAMU, dans le sillage de cette étude, s'engage une réflexion profonde visant une appropriation dès la première année des outils numériques et en particulier du temps réel et de la réalité virtuelle. Cette réflexion s'accompagne d'une démarche de virtualisation des contenus et de la proposition éventuelle d'une formation partiellement virtualisée permettant à des étudiants ne pouvant obtenir de visa de bénéficier du même niveau de formation. L'ensa Nantes a investi quant à elle dans un dispositif immersif de prestige, Coraulis, offrant la possibilité aux étudiants de pouvoir travailler simultanément en analogique et en numérique.

La crise du Covid19 évoqué en début de propos a été aussi un révélateur pour les institutions à basculer rapidement dans une forme de virtualisation des contenus et des protocoles de diffusion. La FAMU ayant déjà repéré les protocoles efficients a été opérationnelle très rapidement, l'ensa Nantes qui n'avait pourtant pas encore engagé cette réflexion a également été réactive grâce à un service informatique extrêmement bien structuré.

Deux notions ont été proposées dans ce texte, la notion d'héritage et la notion d'émergence. Le tableau 1 décrit assez précisément ce que l'on peut identifier dans la notion d'héritage qui embarque la transmission de la pratique architecturale telle qu'opérée depuis l'ouverture des écoles d'architecture. La notion d'émergence que l'on voit 
davantage positionnée dans le tableau 2 décrit non seulement des pratiques nouvelles mais surtout engage quasi mécaniquement une dialectique avec l'environnement social dans lequel s'inscrit le projet. En intégrant dès les phases de conception la gestion des données ou autrement dit la connaissance fine du territoire, on s'impose à intégrer des formes d'objectivation des phénomènes climatiques et sociaux qui seront intégrées dès l'esquisse du projet. L'enjeu s'est donc déplacé de formes de conceptions mettant en concurrence l'outil numérique ou la main en formes de conceptions intégrant un très grand nombre de données inter-agissantes, ce pour quoi, les outils numériques nous sont d'une grande aide.

Pour la pédagogie, les enjeux se déplacent aussi puisqu'au-delà de l'évaluation d'un résultat produit par l'étudiant, l'enseignant doit désormais être particulièrement attentif au processus mis en action par l'étudiant, ce qui permet à ce dernier d'avoir des retours tant sur sa démarche que sur sa production. Cette distinction permet aussi d'identifier de nouvelles formes de pratique de l'architecture, d'inventer ou réinventer le métier de demain.

Nous remercions les collègues qui ont commenté les tableaux afin que nous puissions les améliorer.

\section{Bibliographie}

Coates Nigel, (2012), Narrative Architecture: Architectural Design Primers series, Wiley, Cormerais F., (2015), l'imaginaire en fabrication d'une ville contributive, Nantes, in Poétique du numérique 3, éditions L'Entretemps - Lavérune - 2015, pp 11-14

Coulais J-F., (2014), Images virtuelles et horizons du regard: visibilités calculées dans l'histoire des représentations, MetisPresses

Picon A., (2010), Culture numérique et architecture : une introduction, Birkhauser,

Lescop L., Lu Y. (2018), Les dimensions de la perspective, quand les modes de représentations européennes et chinoises rencontrent l'image numérique. SCAN'18 $8 e$ Séminaire de Conception Architecturale Numérique, Oct 2018, Nantes, France.

Hochscheid E., Halin G., (2018), L'adoption du BIM dans les agences d'architecture en France. SCAN'18 8e Séminaire de Conception Architecturale Numérique, Oct 2018, Nantes, France.

Jabi W., (2013), Parametric Design for Architecture, L. King Publishing,

Kottas D., (2013), Architecture numérique : Nouvelles applications, 2 vol Links Books

Leach N., (2009), Digital Cities AD, Architectural Design July August 2009 Vol. 79, No. 4

Migayrou F., (2003), Architectures non standard, Ed.du Centre Pompidou

Nÿs M., (2018), Architectures en devenir : projeter avec la dimension temporelle des usages. Autour des temporalités de Cedric Price, thèse de doctorat, ensa Versailles et Université de Paris-Saclay, ED SHS pôle SSH.

Schaumann D., Kalay YE, Hong SW, (2017). A Form-Function-Use (FFU) model to simulate human behavior in built environments, EAEA 13: Envisioning Architecture Space/Time/Meaning, Sept 2017, Glasgow, Scotland

Spiller N., (2008), Cybrid (s) Architectures virtuelles, Parenthèses Editions

Spiller N., Visionary Architecture: Blueprints of the Modern Imagination, Thames \& Hudson Ltd, Reprint 2007

Violeau JL, (1999), Quel enseignement pour l'architecture ? Continuités et ouvertures, Editions la Recherche

https://finch3d.com/video-blog/

https://www.darchitectures.com/les-petites-et-moyennes-agences-architecture-menaceesa3879.html

https://www.architectes.org/l-essentiel-des-chiffres-de-la-profession 